\title{
Cyborgs En Centroamérica: Ciberespacio, Políticas Oposicionales Y Pensamiento Feminista Decolonial
}

Reyes-Rivas, Roxana ${ }^{1}$

\section{Resumen}

El contexto centroamericano se caracteriza por una situación periférica tanto en la producción tecnocientífica, como en lo que se refiere al acceso desigual a los productos tecnocientíficos. Esto es particularmente evidente en cuanto a las Tecnologías de la Información y Comunicación (TIC). Se discutirán las características de esta región en lo que concierne al ciberespacio facilitado por la Internet y su apropiación por las mujeres, en particular en grupos organizados. Se utilizará un enfoque teórico decolonial ciberfeminista, pues la región centroamericana no solamente ocupa un lugar periférico dentro del sistema-mundo, sino que, por razones culturales y económicas, el acceso de las mujeres al uso de las tecnologías computacionales e informáticas ha sido aún más restringido, constituyéndolas así en la periferia de la periferia. A través de dicho enfoque se podrá comprender la manera particular en que se utilizan las TIC en nuestra región como instrumento para ejercer sus capacidades y políticas oposicionales que, como diría la pensadora chicana Chela Sandoval, dan pie a conformar el cyborg centroamericano, como expresión de la imaginería propuesta por Haraway (1985). Este enfoque teórico podrá verse funcionando mediante la discusión del ejemplo de un grupo que se opone a situaciones que precarizan la existencia, como el golpe de Estado en Honduras (Feministas en Resistencia).

Ciberespacio. Cyborg Centroamericano. Ciberfeminismo. Decolonialidad. TIC. Internet.

\section{Resumo}

O contexto centroamericano se caracteriza por uma situação periférica tanto na produção tecnocientífica, como no que se refere ao acesso desigual aos produtos tecnocientíficos. Isto é particularmente evidente em relação as Tecnologias de Informação e Comunicação (TIC). Discutiremos as características dessa região no que concerne ao ciberespeaço facilitado pela Internet e sua apropriação pelas mulheres, em particular em grupos organizados. Utilizaremos um enfoque teórico decolonial ciberfeminista, pois a região centroamericana não somente ocupa um lugar periférico dentro do sistema-mundo, mas também, por razões culturais e econômicas, o acesso das mulheres ao uso das tecnologias computacionais e informáticas têm sido ainda mais restringido, contituindo-as assim na periferia da periferia. Através do enfoque apresentado poderemos compreender a maneira particular em que as TIC são utilizadas em nossa região como instrumento para exercer suas capacidades e políticas oposicionais que, como diría a pensadora chicana Chela Sandoval ajudam a admitir o Cyborg centroamericano, como expressão do imaginário proposto por Haraway (1985). Este enfoque teórico poderá ser visto funcionando mediante a discussão do exemplo de um grupo que se opõe a situações que precarizam a existência, como o golpe de estado em Honduras (Feministas em Resistência).

1 Professora do Instituto Tecnológico de Costa Rica Escuela de Ciencias Sociales y Escuela de Computación, Cartago. Costa Rica. 
Ciberespaço. Cyborg Centroamericano. Ciberfeminismo. Decolonialidade. TIC. Internet.

\section{Introducción}

El desarrollo de las Tecnologías de la Información y Comunicación (TIC) en las últimas décadas del S XX, y lo que llevamos del S XXI, ha sido vinculado a una serie de cambios en muchos ámbitos de la vida en las sociedades contemporáneas. En efecto, se ha señalado muchas veces cómo la relevancia que han adquirido estas tecnologías ha propiciado cambios, inclusive, en la economía ${ }^{2}$ (Webster1995/2006), por eso, se le ha llamado "sociedad de la información" (Castro 2006, Webster1995/2006 ) o "Sociedad informacional" (Castells 1996/2005) ${ }^{3}$. Pero, más allá de la caracterización desde la perspectiva estrictamente económica se puede ver que, alrededor de dichas tecnologías, se han gestado formas diferentes de sociabilidad y de producción simbólica, como bien lo han señalado Aronowitz, Martinsons y Mesner. (1998)

Fundamentalmente, la tecnociencia atraviesa la vida contemporánea tanto de las sociedades que se han llamado desarrolladas o metropolitanas, como de aquellas que se han considerado periféricas, subdesarrolladas o en vías de desarrollo. Como lo ha señalado Jesús Martín-Barbero (2010, p. 150) ésta se ha vuelto estructural en las sociedades contemporáneas, dado que introduce "nuevos modos de percepción y de lenguaje (...) nuevas sensibilidades y escrituras".

No obstante, la producción tecnocientífica principalmente ha irradiado desde los grandes centros metropolitanos hacia la periferia. En efecto, desde la Revolución Industrial, la producción científico-técnica de los países del Norte de Europa y, posteriormente, de la América anglosajona, ha marcado la pauta tanto de la producción de conocimiento y tecnociencia, así como del consumo y uso que se le da a estas producciones.

Pero estas relaciones de periferia y metrópoli arriba mencionadas tienen raíces en un

2 Aquí no estoy apelando a un determinismo tecnológico, ya que precisamente haré uso de la categoría de tecnociencia para referirme a una institución social que se encuentra en relación con otras prácticas y productos de la sociedad con las que interactúa muchas veces a manera de vasos comunicantes. (Aronowitz et al. 1998: 22 y sgs.). Esto es, el vínculo cada vez más estrecho entre ciencia y tecnología, el hecho de que éstas cada vez se convierten en un insumo una de la otra y el interés económico y estratégico que importan, nos permite hablar de tecnociencia. Además, la separación categorial de la producción de conocimiento, por un lado y, por otro, de recursos e instrumentos en ciencia y tecnología respectivamente, pierde sentido en el discurso de las humanidades y ciencias sociales contemporáneas. Esto porque cada vez es más evidente el carácter constitutivo mutuo entre la instrumentalidad (tecnología) y el conocimiento (ciencia). (Martín-Barbero 2010; Haraway 1997, 1985/1995).

3 En este documento, siguiendo a Castells, usaremos "sociedad informacional", pues caracteriza mejor el fenómeno, a saber, las tecnologías desarrolladas alrededor del almacenaje, transmisión y procesamiento de información. 
orden colonial precedente. Es decir, hay una serie de relaciones heredadas del colonialismo aun en nuestros días. Éstas son parte de lo que ha sido llamado "colonialidad" o "decolonialidad". De acuerdo con Aníbal Quijano (1993 y 2000) la colonialidad es un conjunto de características y vestigios del poder y la dominación coloniales que hoy día siguen teniendo las sociedades que alguna vez fueron colonias. No obstante, este estado de cosas cobra sentido al ocupar un lugar dentro de un sistema global de control del trabajo, distribución y posesión de la riqueza o lo que, siguiendo a Immanuel Wallerstein (1974), se ha llamado sistema-mundo. En términos de Santiago Castro-Gómez y Ramón Grosfoguel (2007: 13) este término se define como sigue, $p$.

El concepto 'decolonialidad' (...) resulta útil para trascender la suposición de ciertos discursos académicos y políticos, según la cual, con el fin de las administraciones coloniales y la formación de los Estados-nación en la periferia, vivimos ahora en un mundo descolonizado y poscolonial. Nosotros partimos, en cambio, del supuesto de que la división internacional del trabajo entre centros y periferias, así como la jerarquización étnico-racial de las poblaciones, formada durante varios siglos de expansión colonial europea, no se transformó significativamente con el fin del colonialismo y la formación de los Estados-nación en la periferia. Asistimos, más bien, a una transición del colonialismo moderno a la colonialidad global, proceso que ciertamente ha transformado las formas de dominación desplegadas por la modernidad, pero no la estructura de las relaciones centro-periferia a escala mundial.

Así pues, cabe preguntarse cómo se puede entender la sociedad informacional en sociedades periféricas ${ }^{4}$. En otras palabras, ¿cabe hablar de una sociedad informacional desde la periferia? ¿hay metrópolis y periferias dentro de las sociedades latinoamericanas desde la perspectiva de la sociedad informacional? Y si es así, ¿qué retos epistemológicos supone hablar de la sociedad informacional en América Latina ${ }^{5}$ y desde América Latina?

Más aun, tomando en cuenta el uso del ciberespacio y las posibilidades de sociabilidad y producción simbólica que abre ¿podemos pensar en posibilidades particulares en cuanto a producción simbólica y sociabilidad desde América Latina y en particular desde

\footnotetext{
4 No me estoy refiriendo aquí a una localización estrictamente geográfica, sino geopolítica. Dado esto, podemos también hablar de periferias y metrópolis dentro de un mismo ámbito geográfico, inclusive dentro de un mismo país.

5 Siguiendo a Walter Mignolo (2005/2007) se entiende aquí que el nombre "América Latina" es un producto de la matriz epistémica y simbólica de la colonialidad en que vive nuestra región. No obstante, a lo largo de este documento lo seguiré usando por varias razones: 1. este estudio se refiere básicamente a Centroamérica, por lo que no parece procedente llamarle "Abya Yala", como es la tendencia en los países andinos; 2. el interés de este estudio es cómo se enmarca nuestra región en el uso de las TIC y cómo la producción tecnocientífica nos enmarca precisamente como "América Latina".
} 
Centroamérica? En otras palabras, a pesar de la supuesta transnacionalidad que se entiende como propia de los usos de las TIC (ciberespacio), ciertamente hay condiciones materiales dentro del subcontinente latinoamericano que no pueden ser obviadas a favor del acceso.

Algunos sectores de las sociedades latinoamericanas que han obtenido acceso a las tecnologías de la información y el capitalismo en nuestro subcontinente también han apostado por la producción de bienes culturales a través de las TIC (Castro-Gómez). No obstante, la brecha digital es amplia (Martín-Barbero 2010, p. 156) y las condiciones precarias de supervivencia, así como un repunte del autoritarismo y la violencia de estado contra las aspiraciones democráticas de los pueblos, son condiciones bajo las cuales un número considerable de personas, en especial en Centro América, tienen que vérselas cotidianamente.

En efecto, en el ámbito cultural es donde más evidente se hace uso de la tecnología para la circulación de la información (Webster 1995/2006, p. 19 y sgs.). Pero más allá de la producción de bienes culturales que ha sido potenciada por las TIC, en tanto que actividad económica, el entorno ciberespacial o espacio virtual también posibilita la redefinición de la manera en que se suscita la producción simbólica. En otras palabras, con el desarrollo de una vivencia cada vez más constante y un uso más diverso del ciberespacio se forma, a su vez, cibercultura o ciberculturas. Aparecen diferentes formas de expresión artística, formas diferentes de interacción y de identificación.

Dentro de este marco, quiero discutir el uso de las TIC en Centro América. Aunque estas tecnologías, en términos de producción y cultura, marcan una gran diferencia entre las áreas metropolitanas (Norte global) y países periféricos (Sur global), el uso de estas tecnologías se ha vuelto muy importante para la resistencia política en el Sur global. El ciberespacio se ha convertido en un recurso de resistencia para grupos de mujeres que luchan contra la inestabilidad, desigualdad política y en pro de los Derechos Humanos en Centro América. Esto me parece especialmente importante dado que por razones culturales y económicas, las mujeres tienen una posición periférica con respecto al acceso y uso de las $\mathrm{TIC}^{6}$.

6 De acuerdo con informes de la CEPAL (2013: 40-41) las mujeres acceden menos a Internet debido a que acceden menos al trabajo remunerado, siendo su taza de pobreza mayor, aunque aquellas que están empleadas de forma remunerada son usuarias muy eficientes. 
Mediante la combinación de los enfoques de la colonialidad y el ciberfeminismo, en este trabajo quiero discutir y analizar cómo algunas mujeres en la región construyen su propia agencia a través de formas creativas de uso y de hacer red dentro del ciberespacio.

\section{Ciberfeminismo y cyborg centroamericano}

Las pensadoras ciberfeministas del Norte global como Rossi Braidotti (1996 y 1994/2000) (filosofía del como-si) y Donna Haraway (1985/1995) (imaginería cyborg) ${ }^{7}$, han prestado particular atención a la resistencia en el ciberespacio y la informática especialmente mediante una respuesta estética ${ }^{8}$. El ciberfeminismo se ha caracterizado como una demanda por el derecho a utilizar las TIC y el ciberespacio de forma crítica ante las dinámicas patriarcales y machistas que ahí se suscitan. Braidotti (1996) lo expresa de la siguiente manera:

La estrategia más efectiva para las mujeres sigue siendo utilizar la tecnología para liberar nuestra imaginación colectiva del falo y sus valores accesorios como son el dinero, la exclusión y la dominación, el nacionalismo, la femineidad icónica y la violencia sistematizada.

A su vez, la teórica chicana Chela Sandoval sostiene que:

"la consciencia cyborg puede ser entendida como la encarnación tecnológica de una forma particular y específica de consciencia oposicional que he descrito en otra parte como "feminismo del tercer mundo en Estados Unidos (U.S. third world feminism)".9 (1995, p. 408, Trad. RRR)

Algunos grupos de mujeres centroamericanas -y de otras partes del Sur global- van

7 ...De acuerdo con el "Manifiesto cyborg"... poco a poco todos nos vamos convirtiendo en cyborgs pues la tecnociencia contemporánea ha traspasado los límites entre lo que tradicionalmente hemos considerado lo natural y lo artificial. Más aún, las distinciones entre animal, humano y máquina se han vuelto borrosas, a través de tecnologías como el ADN recombinante, los implantes electrónicos y la ubicuidad de las TIC y, hoy podríamos agregar, las tecnologías digitales. Esto ha sido posible gracias a la teoría cibernética donde todo, desde un termostato hasta un ecosistema, pueden ser vistos como sistemas que permiten la circulación de información que, a su vez, los retroalimenta y los ajusta. Haraway convierte lo cyborg en un elemento capaz de desafiar las estructuras capitalistas, patriarcales y militaristas, aunque venga de ellas, siendo el germen de la destrucción del capitalismo tardío. Esto pues, llevará a que en las políticas cyborgs las gentes no se busquen, ni se rebelen por identificaciones esencialistas y rígidas, sino que, al igual que en los sistemas cibernéticos, estableciendo redes de comunicación y retroalimentación, a través de coaliciones por afinidad, no por identidad.

8 Braidotti, a su vez, usa como ejemplos de su "filosofía del como-si" a artistas como Laurie Anderson, Nell Tenhaff, Barbara Kruger y Cindy Sherman. (Aunque no es el interés de este trabajo, en América Latina, dentro de esta línea podríamos citar a La Torta Golosa y Liliana Felipe).

9 “...los pueblos oposicionales colonizados de las Américas ya han desarrollado las destrezas cyborg que se requieren para la supervivencia bajo las condiciones tecno-humanas como requisito para la supervivencia bajo el dominio sufrido durante los últimos trescientos años. Es interesante, sin embargo, que los teóricos de la globalización se ocupan de la introducción de una política oposicional "cyborg" como si estas políticas hubieran emergido con el advenimiento de la tecnología electrónica únicamente y no como un requerimiento de la consciencia en oposición que se desarrolló bajo formas previas de dominación". (408)(Trad. RRR) 
más allá y no buscan solamente la respuesta estética estilizada, sino también acciones efectivas a partir del establecimiento de redes ciberespaciales, como veremos más adelante. Esto es, si bien en América Latina -y en particular en Centro América- se produce poca tecnología de punta -y la mayoría de la que se ha producido corresponde a lo que he llamado "maquila tecnológica"10, las TIC también juegan un papel importante en algunos grupos de nuestra región. Como lo reconoce la activista hondureña Daisy Flores:

Los movimientos de mujeres y feministas hemos apuntado que la tecnología tiene un valor político y social, porque su contribución a la construcción colectiva de la vida puede ser una herramienta tanto de empoderamiento como de perpetración de las desigualdades en general y en particular de las de género. (2012).

Así pues, siguiendo a nuestras ciberfeministas, lo cyborg centroamericano proviene no solamente del uso de las TIC para la política oposicional, sino de la consciencia de su carácter de herramienta y de prótesis de las políticas oposicionales con las cuales, de antemano, los grupos en resistencia se han preparado desde la colonia y la colonialidad.

\section{Desafíos epistemológicos}

El uso del ciberespacio pide también una revisión epistemológica. En efecto, la desterritorialización del espacio que esto conlleva, nos permite formar comunidades, identidades y sobretodo, alteridades de maneras muy diferentes que las que suscitan los límites geográficos que ha requerido tradicionalmente la interacción. En palabras de Bermúdez y Martínez:

Las NTIC[sic ${ }^{11}$ impulsan un carácter global diferente al que conocimos a través de los modos tecnológicos tradicionales de transporte y comunicación, en donde la movilización de objetos y sujetos estaban circunscritos a los marcos definidos por los límites de un Estado nación. Las NTIC desbordan los límites jurídicos, políticos y territoriales del Estado nación y relativizan la distinción entro lo próximo y lo lejano, acerca las distancias y desdibuja las fronteras de las culturas nacionales. Al usar el término "desanclaje" de

\footnotetext{
10 Por ejemplo, en Costa Rica, como parte de Centro América, estaría incluida no solamente la empresa Intel, sino también AdAstra, subsidiaria de una empresa estadounidense que investiga sobre la construcción de un motor de plasma y sistemas de energía renovable (http://www.adastrarocket.com/aarc/es). Si bien ambas empresas tienen divisiones de investigación y desarrollo, el peso fundamental de Intel ha estado en prueba y ensamblaje. De hecho, cuando se anunció a mediados de 2014 que esta última división se retiraba de Intel Costa Rica, esto fue percibido como una pérdida sensible, a pesar de que la división de investigación y desarrollo se mantuvo.
}

11 ... Nuevas Tecnologías de la Información y la Comunicación. Si bien en el texto citado se usa dicho término, en el cuerpo de este artículo se usa el término más comúnmente utilizado "Tecnologías de la Información y la Comunicación" abreviado por "TIC". Más abajo veremos que Leah Lievrow (2011) argumenta que dichas tecnologías siempre son nuevas. 
Giddens (1990), podemos decir que la cultura nacional pierde en el ciberespacio el lazo orgánico con el territorio y con la lengua y, de hecho, se alteran las formas tradicionales de entender las identidades nacionales. (p.1314)

Castells (1996/2005) ha abundado en la alteración o abandono del tiempo secuencial dentro del mundo virtual. Esto permite que los sujetos se pluralicen y se alejen del espacio exterior o concreto. Esto pues, implica que una serie de producciones simbólicas no son abarcables con las categorías convencionales de los estudios culturales, ya que parecieran desaparecer las identidades nacionales, el sentido de comunidad convencional ${ }^{12}$. Además, la construcción del Otro opera de maneras diferentes en el ciberespacio, donde la interacción cara a cara pierde importancia, aunque no totalmente. Ahora bien, aunque la sociabilidad ciberespacial adquiere un carácter transnacional o desterritorializado, esto no quiere decir que sea un mundo totalmente separado de lo local; por el contrario, tiene efectos y retroalimentaciones desde el espacio local y hacia el espacio local.

Cabe aquí pensar en la categoría de hibridación13. En efecto, estamos ante un proceso de hibridación de la cultura misma a través de esos mismos intercambios de la sociabilidad en el espacio newtoniano retroalimentado por el ciberespacio y viceversa. Aquí me refiero al espacio material u offline como "newtoniano", tomando en cuenta que el espacio donde tradicionalmente nos movemos es el que en la Modernidad ha sido descrito por la física newtoniana. En otras palabras, el uso de ese término hace énfasis en que nuestra cotidianidad contemporánea acaece en dos espacios uno fundado y fundamentado por la Modernidad y otro que interactúa con dicho espacio y que desestabiliza el marco de referencias y el sistema de coordenadas en que suponemos transcurre el mundo de la vida.

Leah Lievrouw (2011) señala otro proceso de hibridación, a saber, el de reconfiguración. Este alude al carácter recombinante de lo que ella llama las "nuevas

\footnotetext{
12 Tomás Maldonado (1998) habla de la fragmentación del yo. Este mismo autor hace una descripción y diferenciación entre lo que él llama comunidades fuertes o reales y comunidades débiles o virtuales.

13 " entiendo por hibridación procesos socioculturales en los que estructuras o prácticas discretas, que existían en forma separada, se combinan para generar nuevas estructuras, objetos y prácticas. A su vez, cabe aclarar que las estructuras llamadas discretas fueron resultado de hibridaciones, por lo cual no pueden ser consideradas fuentes puras". (García CancliniOp. Cit.: 14) El concepto de hibridación ha tenido varios tratamientos en diferentes ámbitos y corrientes de pensamiento académico (Kraniauskas 2000), la de García Canclini es la que ha cobrado más relevancia en Latinoamérica y la que parece más adecuada para estudiar el asunto aquí planteado.
} 
tecnologías de la comunicación".14 De esta forma, la reconfiguración la podemos ver en el uso de TIC ideadas con diferentes fines que se adoptan de forma inesperada (p.e., el uso de YouTube como medio alternativo para transmisión de noticias). Lo particular de esta comprensión del término consiste en que las nuevas tecnologías de comunicación no se usan para representar una agenda determinada y ya existente, sino que "ellas mismas son arenas y espacios de relaciones y acción, y herramientas para la intervención (...), que son continuamente reorganizadas y reconfiguradas conforme cambian las prioridades de los movimientos" (Lievrouw 2011, p. 217).

Dado que el interés está puesto en la dimensión social y cultural de esta tecnología, no en la propiamente técnica, la categoría de ciberespacio viene a cuento, pues este es posible solamente como hibridación entre humanos y máquinas. Como bien lo explica Joan Mayans (2002):

El ciberespacio no es la suma de ordenadores, líneas telefónicas, satélites,
teléfonos móviles y el largo etcétera de máquinas que se le quiera sumar. (...)
La idea de ciberespacio nos remite a eso que ocurre en su interior. O a
aquello que ocurre por medio de todas esas máquinas y dispositivos. No
obstante, todo eso ocurre en tanto que producido socialmente, por seres
humanos. Así, el ciberespacio no está habitado por las máquinas, sino
posibilitado por éstas. (237).

Por otra parte, debido a que el ciberespacio no es la suma de un conjunto de dispositivos, sino la interacción de humanos a través de dicho conjunto de dispositivos, éste solamente existe en tanto que es ocupado por los usuarios de las tecnologías pertinentes, es decir, como espacio practicado. En palabras de Michel de Certeau el espacio practicado:

Existe cuando se toman en consideración los vectores de dirección, las velocidades y las variables temporales. Así, el espacio se compone de intersecciones de elementos en movimiento (...). El espacio ocurre como el efecto producido por las operaciones que lo orientan, lo sitúan, lo temporalizan, y lo hacen funcionar (...). Desde este punto de vista, en relación a los sitios/lugares [places] (sic), el espacio es como la palabra cuando es pronunciada (...) Así, la calle definida geométricamente por los planificadores urbanos es convertida en espacio por los caminantes. (Citado en Mayans Op. Cit., 240)

\section{Un ejemplo}

Para terminar, quiero discutir un ejemplos del uso del ciberespacio como ámbito de

14 De acuerdo con Lievrouw, precisamente ese carácter recombinante es lo que las hace siempre nuevas. 
resistencia, a saber, las Feministas en Resistencia. Para caracterizar a este grupo se utilizarán las categorías propuestas por Lievrouw (2011, p. 215): género (genre)15 y sus tres dimensiones, a saber, alcance (scope), posicionamiento (stance) y agencia (action o agency). En tanto género, las Feministas en Resistencia se constituyen en parte de un periodismo participatorio y movilización mediada. Cabe destacar que éste promueve un proyecto de conocimiento comunal (commons knowledge).

De acuerdo con las corrientes teóricas y conceptos arriba planteados podemos plantear la mediación como concepto sombrilla que cubre el marco analítico y enfoque teórico de este trabajo. Basada en Lievrouw la mediación sirve para comprender cómo la reconfiguración de sistemas tecnológicos y la remediatización de la expresión, interacción, relaciones y estructuras sociales y contenido, son procesos emergentes que se condicionan mutuamente. En sus propias palabras:

...[la] mediación comprende una continua, mutuamente reconfigurante relación entre los usos que la gente hace de las tecnologías de la comunicación (reconfiguración) y su acción comunicativa (remediatización) que produce cambio social y tecnológico. (231 Trad. RRR).

De acuerdo con Martín-Barbero (2010,p.148 y sgs.) la tecnicidad contemporánea ha constituido un ecosistema comunicacional y, por eso mismo, se torna estructural. Debido a esto, la tecnicidad es una de las formas que toma la mediación que permitirá comprender las relaciones arriba apuntadas entre reconfiguración y remediatización.

\section{Conclusiones}

Como se puede ver, se ha presentado una lectura decolonial, pues se concentra en entender cómo funciona el uso de las TIC y la sociedad informacional en ámbitos como el centroamericano, más allá de los negocios y la academia. En efecto, a dicha lectura le viene la decolonialidad de comprender qué significa la sociedad informacional en una región periférica, receptora de las TIC, más que productora.

En este caso, hemos visto que hay formas propias del ciberfeminismo en ámbitos periféricos que no obedecen a la imagen más común de lo que se considera ciberfeminismo,

15 Esta autora enumera los siguientes cinco géneros de nuevos medios alternativos y de activismo: disrupción cultural (Culture jamming), computación alternativa (Alternative computing), periodismo participativo (Participatory journalism), movilización mediatizada (Mediated movilization), conocimiento comunal (Commons knowledge). 
sobretodo en los ámbitos académicos metropolitanos, aunque Haraway ya lo previó. Así pues, grupos de feministas en la región centroamericana han tomado conciencia de la importancia del uso de las TIC, en aras de agilizar sus políticas oposicionales. Esto ya no solamente en la lucha por las demandas específicamente feministas, sino que, en el caso de las Feministas en Resistencia, para oponerse a situaciones políticas que ponen en condiciones de gran precariedad a la población en general.

Así pues, lo cyborg en Centroamérica empata con lo que menciona Sandoval, a saber, que en las regiones periféricas, para cuando las TIC se convierten en tecnologías fundantes de una forma diferente de sociabilidad y producción, ya grandes grupos de la población habían desarrollado las capacidades de supervivencia y resistencia, de tal forma que lo cyborg y el ciberfeminismo no son más que la expresión tecnológica contemporánea de las políticas oposicionales que han tenido que desarrollar los pueblos de nuestra región desde la colonia.

\section{Referencias}

Aronowitz, S., Martinsons, B. y Mesner, M. (comps.). Tecnociencia y cibercultura. La interrelación entre cultura, tecnología y ciencia. Traducción de Patrick Ducher. Barcelona: Editorial Paidós, 1998.

Braidotti, Rosi. Sujetos nómades. Corporización y diferencia sexual en la teoría feminista contemporánea. Traducción de Alcira Bixio. Buenos Aires: Paidós.1994/2000.

"Un ciberfeminismo diferente". En, textos online sobre arte y mujer. Traducción de Carolina Díaz. Recuperado de http://www.estudiosonline.net/texts/diferente.html, 1996.

Castells, Manuel. La sociedad red. La era de la información: Economía, sociedad y cultura. Traducción de Carmen Martínez Gimeno. (Sexta Edición) México: Siglo Veintiuno Editores. Vol. I, 1996/2005

. El poder de la identidad. La era de la información: Economía, sociedad y cultura. Traducción de Carmen Martínez Gimeno. (Cuarta Edición) México: Siglo Veintiuno Editores. Vol. II. 1998/2003. . et. al. La transición a la sociedad red. Barcelona: Ariel,2007.

Castro Bonilla, Alejandra. Derechos de autor y nuevas tecnologías. San José: EUNED, 2006. Castro-Gómez, Santiago y Ramón Grosfoguel (eds.). El giro decolonial. Reflexiones para una diversidad epistémica más allá del capitalismo global. Bogotá: Siglo del Hombre Editores, 02, dez, 2016.

Comisión Económica para América Latina y el Caribe (CEPAL) Mujeres en la economía digital. Superar el umbral de la desigualdad, Santo Domingo, 2013.

Flores, Daisy. "Reflexiones sobre...Descubriendo mundos y compartiendo resistencias en línea." Boletín de GenderlT.org (Abril 2012) Recuperado de http://www.genderit.org/es/node/3545/ mayo, 2014. 
García Canclini, Nestor. "Las culturas híbridas en tiempos globalizados”, en Culturas híbridas. Estrategias para entrar y salir de la modernidad. (Primera reimpresión) Buenos Aires: Editorial Paidós SAICF, 2005.

Haraway, Donna J. "A Cyborg Manifesto: Science, Technology, and Socialist Feminism in Late Twentieth Century". En Simians, Cyborgs and Women. The Reinvention of Nature New York: Routledge.. 1985/1991 p149-181.

.. "Manifiesto para cyborgs: ciencia, tecnología y feminismo socialista a finales del siglo XX". En Ciencia, cyborgs y mujeres. La reinvención de la naturaleza. (251-311) Traducción de Manuel Talens. Madrid: Ediciones Cátedra, S.A., 1985/1995

..Modest_Witness @ Second_Millennium.FemaleManMeets_OncoMouse. Feminism and Technoscience. New York: Routledge, 1997.

Kraniauskas, John. "Hybridity in a transnational frame: Latin-Americanist and post-colonial perspectives on cultural studies". En Avtar Bran y Annie E. Coombes (eds.) Hybridity and its Discontents. PoliTIC, Science, Culture. (235-256) New York: Routledge, 2000.

Lievrouw, Leah A. Alternative and Activist New Media. Cambridge, UK: Polity Press,2011.

Maldonado, Tomás. Crítica de la Razón Informática. Traducción de Juan Carlos Gentile Vitale. Barcelona: Ediciones Paidós Ibérica, 1998.

Martín-Barbero, Jesús. De los medios a las mediaciones. Comunicación, cultura y hegemonía. (Tercera edición) México: Editorial Gustavo Gili, 1993.

. "Convergencia digital y diversidad cultural". En Moraes, Dênis de (Ed.) Mutaciones de lo visible. Comunicación y procesos culturales en la era digital. (137-165) Buenos Aires: Editorial Paidós SAICF, 2010.

Mayans i Planells, Joan. Género Chat. $O$ cómo la etnografía puso un pie en el ciberespacio. Barcelona: Editorial Gedisa S.A., 2002.

Mignolo, Walter D. La idea de América Latina. La herida colonial y la opción decolonial. Traducción de Silvia Jawerbaum y Julieta Barba. Barcelona: Gedisa Editorial, 2005/2007.

Quijano, Aníbal "Colonialidad del poder, eurocentrismo y América Latina". En Edgardo Lander (Ed.) La colonialidad del saber: eurocentrismo y ciencias sociales. Perspectivas latinoamericanas. (201-246) Buenos Aires: CLACSO, febrero de 2000. 2000.

Colonialidad del poder y clasificación social". Journal of World-Systems Research, XI(2),

Sandoval, Chela. "New Sciences: Cyborg Feminism and the Methodology of the Oppressed". En Chris Hables Gray (Ed.) (1995) The Cyborg Handbook. (407-421) New York: Routledge, 1995. 